\title{
Fatores de risco para anemia em crianças de 6 a 12 meses no Brasil
}

\author{
Mônica Glória Neumann Spinelli, ${ }^{1}$ Dirce Maria Lobo Marchioni, ${ }^{1}$ \\ José Maria Pacheco Souza, ${ }^{1}$ Sonia Buongermino de Souza ${ }^{1}$ \\ e Sophia Cornbluth Szarfarc ${ }^{1}$
}

Como citar Spinelli MGN, Marchioni DML, Souza JMP, Souza SB de, Szarfarc SC. Fatores de risco para anemia em crianças de 6 a 12 meses no Brasil. Rev Panam Salud Publica. 2005;17(2):84-91.

RESUMO Objetivo. Estimar a prevalência de anemia e investigar os fatores de risco a ela associados em lactentes brasileiros que recebem atendimento na rede básica de saúde.

Método. Estudo transversal com 2715 crianças entre 6 e 12 meses de idade, residentes em 12 municípios das cinco regiões do Brasil. As mães ou responsáveis responderam a um questionário para coleta de informações sobre a criança e sua alimentação. Foram medidos o peso e a altura para determinação do estado nutricional. Também foi feita a dosagem da concentração de hemoglobina com fotômetro portátil HemoCue, considerando-se como anemia o nível $<11 \mathrm{~g} /$ dL. A prática alimentar considerou a situação das crianças no momento do estudo (current status). A associação entre as variáveis e a anemia foi verificada inicialmente por análise bivariada e posteriormente por regressão logística múltipla segundo modelo hierarquizado.

Resultados. A prevalência média de anemia para todo o grupo foi de 65,4\%. A análise múltipla identificou os seguintes fatores de risco para anemia: morar na Região Sudeste [razão de produtos cruzados, ou OR, de 1,57 (1,25 a 1,99)], idade materna inferior a 20 anos [OR = 1,58 (1,21 a 2,07)], peso ao nascer < $2500 \mathrm{~g}[\mathrm{OR}=2,09$ (1,48 a 2,95)], não receber leite materno $[O R=1,28(1,02$ a 1,61)] ou estar em aleitamento misto $[O R=1,40(1,10$ a 1,78)] e sexo masculino $[O R=1,24(1,06$ a 1,46)].

Conclusões. A alta proporção de crianças anêmicas indica a necessidade de enfatizar, nos programas de pré-natal e puericultura do país, medidas de intervenção e controle desse distúrbio nutricional. Os presentes resultados podem orientar tais ações, que devem privilegiar os grupos de maior risco, como os bebês de baixo peso e os filhos de mães adolescentes.

Palavras-chave Anemia ferropriva, nutrição do lactente, nutrição da criança, fatores de risco, estudos epidemiológicos.

A anemia ferropriva acomete cerca de 2 bilhões de habitantes no mundo todo e vem aumentando nas últimas

\footnotetext{
Universidade de São Paulo (USP), Departamento de Nutrição. Correspondência para Mônica Glória Neumann Spinelli no seguinte endereço: Rua Itacolomi 293 apto. 81, Higienópolis, CEP 01239-020, São Paulo, SP, Brasil. E-mail: spinelli@usp.br
}

décadas (1). É causada por um desequilíbrio entre a quantidade de ferro biologicamente disponível e a sua necessidade orgânica (2). No Brasil, a anemia ferropriva foi apontada em 1997 como a segunda maior deficiência causadora de doenças na infância (3); em todo o mundo, ela está sendo considerada como um dos grandes proble- mas de saúde pública. Crianças e gestantes constituem os grupos mais vulneráveis a esse tipo de anemia, em virtude do aumento das necessidades de ferro, induzido pela rápida expansão da massa celular vermelha e pelo crescimento acentuado dos tecidos (4).

A anemia ferropriva é determinada por um grande número de co-variáveis 
relacionadas a condições socioeconômicas, ambientais e nutricionais, o que dificulta o estudo dos fatores de risco (5-7). Para esse tipo de estudo epidemiológico, a modelagem hierarquizada é uma alternativa aplicável, pois permite a seleção das variáveis mais fortemente associadas à ocorrência do evento (8).

No Brasil, apesar de vários trabalhos de detecção de prevalência terem mostrado uma situação bastante preocupante em todo o país, não há pesquisas de abrangência nacional sobre a anemia ferropriva. Assim, o presente artigo se propõe a estimar a prevalência e analisar os fatores de risco para anemia em crianças com idade entre 6 meses e 1 ano, utilizando um modelo com seleção hierárquica em uma amostra de crianças das cinco regiões geográficas brasileiras.

\section{MATERIAIS E MÉTODOS}

O presente artigo descreve um estudo transversal realizado em 12 centros urbanos nas cinco regiões geográficas do Brasil: Maringá (Estado do Paraná) e Porto Alegre (Rio Grande do Sul), na Região Sul; Viçosa (Minas Gerais), Rio de Janeiro (Estado do Rio de Janeiro) e Santo André e São Paulo (Estado de São Paulo), no Sudeste; Cuiabá, (Mato Grosso) e Brasília (Distrito Federal), no Centro-Oeste; Teresina, (Piauí) e Salvador (Bahia), no Nordeste; Rio Branco (Acre) e Manaus (Amazonas), no Norte.

A Região Sul é a menor região brasileira, porém é a segunda região mais industrializada do país, possuindo também uma agricultura bem desenvolvida. A expectativa de vida é de 65 anos para os homens e 72,5 anos para as mulheres, e a taxa de analfabetismo é de $18 \%$. A Região Sudeste é a mais desenvolvida, com a maior população (densidade demográfica de 78,09 habitantes por $\mathrm{km}^{2}$ ), maior índice de urbanização e maior parque industrial (49\% das indústrias do país). Tem uma agropecuária de peso na economia do país e o maior e mais diversificado setor terciário. A expectativa de vida é de 63,5 anos para os homens e 71,6 anos para as mulheres, e a taxa de analfabetismo é de $18 \%$.

No Centro-Oeste está localizada Brasília, capital do país. Com clima tropical e semi-úmido, a região é produtora de soja, trigo, algodão, feijão, arroz e carne. A atividade industrial é pobre. A expectativa de vida para os homens é de 64,3 anos e, para as mulheres, de 71,5 anos. A taxa de analfabetismo é de $20 \%$. A Região Nordeste tem clima tropical úmido na faixa litorânea e semi-árido no interior. A economia da região se baseia na agricultura açucareira e cacaueira e também na exploração do petróleo no litoral e na plataforma continental. A expectativa de vida para os homens é de 60,8 anos e, para as mulheres, de 67,7 anos. O índice de analfabetismo é de $41 \%$. Por fim, a Região Norte, a maior região brasileira, é a menos desenvolvida e a menos povoada, com apenas 3,33 habitantes por $\mathrm{km}^{2}$. Possui clima equatorial, e a expectativa de vida é de 63,8 anos para os homens e de 71 anos para as mulheres. A taxa de analfabetismo é de 19\% (9).

A amostra do presente estudo foi constituída por 2715 crianças com idade entre 6 e 12 meses atendidas em unidades básicas municipais de saúde para acompanhamento de rotina do crescimento e realização de vacinas. Optou-se por essa faixa etária pois o critério da Organização Mundial da Saúde (OMS) para determinação de anemia é estabelecido a partir do $6^{\circ}$ mês. O número mínimo da amostra em todas as localidades foi calculado com base em uma estimativa de 50\%.

O estudo foi aprovado pelo Comitê de Ética da Faculdade de Saúde Pública da Universidade de São Paulo (USP), e os dados foram coletados após autorização dos pais.

Os dados foram coletados entre os anos de 1998 e 2000. Em cada município, contou-se com um responsável local, que ficou encarregado do treinamento dos entrevistadores para preenchimento do formulário e coleta das amostras sangüíneas. Esses responsáveis locais foram treinados inicialmente pelos coordenadores do projeto para atuar como multiplicadores e garantir a uniformidade da pesquisa.
Para facilitar o preenchimento do questionário, estava disponível um manual de instruções. A coleta foi feita, em algumas localidades, pelos atendentes de enfermagem do serviço de saúde, e em outras, por alunos, bolsistas ou estagiários.

O período de coleta foi diferente para cada município, variando de 4 meses até mais de 1 ano, e foi determinado pela infra-estrutura do local. Os questionários preenchidos foram revisados e codificados antes do início da digitação, realizada em duplicata. Dependendo da estrutura do centro, os dados foram digitados localmente ou enviados para serem digitados na Faculdade de Saúde Pública da USP.

Os dados de peso e estatura ao nascer, tipo de parto e duração da gestação foram obtidos, quando possível, do cartão da maternidade. Os demais foram obtidos na entrevista com a mãe ou responsável, durante o atendimento na unidade básica de saúde. A dosagem da hemoglobina foi feita utilizando-se fotômetro portátil (HemoCue) para leitura direta em amostras sangüíneas obtidas por punctura do pé, com lancetas descartáveis, recomendado para uso em investigações populacionais sobre prevalência de anemia devido ao pequeno volume de sangue exigido e à imediata obtenção do resultado, que é expresso em g/dL (10-13). Considerou-se anemia uma concentração de hemoglobina inferior a $11 \mathrm{~g} /$ dL, segundo critério da OMS (14).

A prática alimentar foi determinada pelo processo denominado current status, ou seja, considerando a situação da criança — sua idade e relação com o evento de interesse- no momento da pesquisa $(15,16)$. A associação entre anemia e seus possíveis determinantes foi estudada inicialmente por análise bivariada e, posteriormente, por regressão logística múltipla com seleção hierarquizada de variáveis, utilizando-se o programa Stata 8 (17). O conjunto inicial foi composto pelas variáveis que, individualmente, apresentaram um $P$ descritivo do teste de Wald $<0,20$. Na análise múltipla, variáveis que foram significativas na etapa de entrada ( $P$ descritivo $<0,05)$ permaneceram no modelo até o final, mesmo 
FIGURA 1. Modelo conceitual com seleção hierárquica para determinação da prevalência de anemia em crianças de 6 a 12 meses, Brasil, 2000

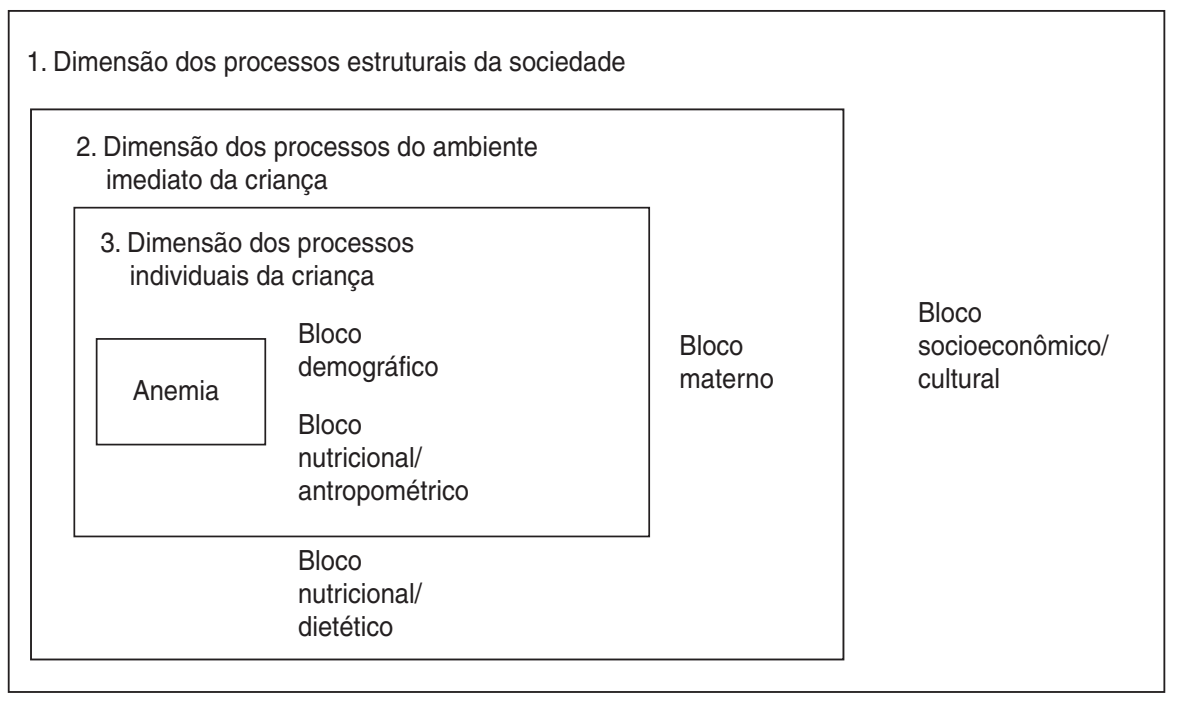

a Adaptado de Silva et al. (6).

que tenham perdido sua significância em etapas posteriores. Os resultados foram expressos pela razão de produtos cruzados (odds ratio, OR) e respectivos intervalos de confiança (IC).

Para a formação do modelo, estruturou-se inicialmente um marco teórico com as variáveis mais freqüentemente descritas na literatura como associadas à anemia, discriminando-as em blocos com seleção hierárquica com base no modelo proposto por Silva et al. (6), que considera três dimensões (figura 1): dimensão dos processos estruturais da sociedade; dimensão dos processos do ambiente imediato da criança; e dimensão dos processos individuais da criança.

Na primeira dimensão foi considerado o bloco socioeconômico/cultural, que congrega as variáveis região (Sul, Sudeste, Centro-Oeste, Nordeste ou Norte) e escolaridade paterna e materna ( $<5$ anos; de 5 a 8 anos; $\geq 9$ anos). Na dimensão dos processos do ambiente imediato da criança foi considerado o bloco materno, que levou em conta as variáveis idade $(<20$ anos ou adolescente; de 20 a 30 anos; $\geq 31$ anos), tempo gestacional ( $<9$ meses ou pré-termo; $\geq 9$ meses ou termo) e tipo de parto (normal, cirúrgico).
Na dimensão dos processos individuais da criança foram considerados três blocos, o demográfico, o nutricional/antropométrico e o nutricional/dietético. Fizeram parte do bloco demográfico as variáveis sexo, idade (6 a 7 meses; 8 a 9 meses; 10 a 11 meses) e peso ao nascer $(<2500 \mathrm{~g}$ ou baixo peso; $\geq 2500 \mathrm{~g}$ ). No bloco nutricional/antropométrico, o índice utilizado para avaliar o estado nutricional das crianças foi o escore $Z$ de peso para idade, tendo como referência o padrão norte-americano (National Center for Health Statistics, NCHS). Os pontos de corte foram: escore $Z$ de peso para idade $<-1$; escore $Z$ de peso para idade de -1 a +1 ; e escore $Z$ de peso para idade $>1$ desvio padrão $( \pm 1$ escore $Z)$ abaixo da mediana do ín-dice antropométrico da população de referência.

Fizeram parte do bloco nutricional/ dietético as seguintes variáveis:

- ter sido amamentado (variável dicotômica一sim/não);

- situação atual de aleitamento: aleitamento materno complementado; aleitamento materno exclusivo ou predominante; aleitamento misto; sem aleitamento materno;
- consumo de alimentos ricos em ferro: dieta com carnes e com feijão; sem carnes e sem feijão; com carnes e sem feijão; sem carnes e com feijão.

\section{RESULTADOS}

A prevalência média de anemia (hemoglobina $<11 \mathrm{~g} / \mathrm{dL}$ ) entre as 2715 crianças do estudo foi de $65,4 \%$. As crianças diagnosticadas como anêmicas foram encaminhadas para tratamento. A tabela 1 mostra os resultados da análise bivariada para a prevalência de anemia no $2^{\circ}$ semestre de vida. A anemia esteve significativamente associada $(P<0,20)$ com a região de moradia, a escolaridade materna, a idade materna, o tempo de gestação, o peso ao nascer, o estado nutricional, o sexo, a situação atual de aleitamento e o consumo de alimentos com ferro. A anemia foi maior nos meninos $(67,6 \%)$, com uma associação estatisticamente significativa $(P=0,018)$ (tabela 1$)$.

Na regressão logística múltipla (tabela 2), a primeira variável selecionada para entrar no modelo foi a região. A Região Sul foi a de menor risco para anemia, enquanto o Sudeste foi a região de maior risco, tomando o Sul como referência. Na segunda etapa, a escolaridade materna entrou no modelo por haver se apresentado significativa na análise bivariada, condição essa que não se manteve, sendo excluída da etapa seguinte. Na terceira etapa, todas as variáveis (região, idade materna e tempo de gestação) mantiveram sua significância. A Região Sudeste e a idade materna menor do que 20 anos representaram fatores de risco para anemia quando comparados aos valores de referência, a Região Sul e a idade materna $\geq 31$.

Na quarta etapa foi detectado risco para baixo peso ao nascer. $O$ peso inferior a $2500 \mathrm{~g}$ no nascimento duplicou a probabilidade de anemia após o $6^{\circ}$ mês $(\mathrm{OR}=2,09)$. Quanto ao tipo de aleitamento, o risco foi maior para as crianças com aleitamento artificial e misto do que para as crianças que recebiam exclusivamente leite materno. O consumo de carnes representou um fator de proteção (tabela 2). Na quinta 
TABELA 1. Prevalência de anemia em crianças de 6 a 12 meses conforme variáveis selecionadas e análise bivariada, Brasil, 2000

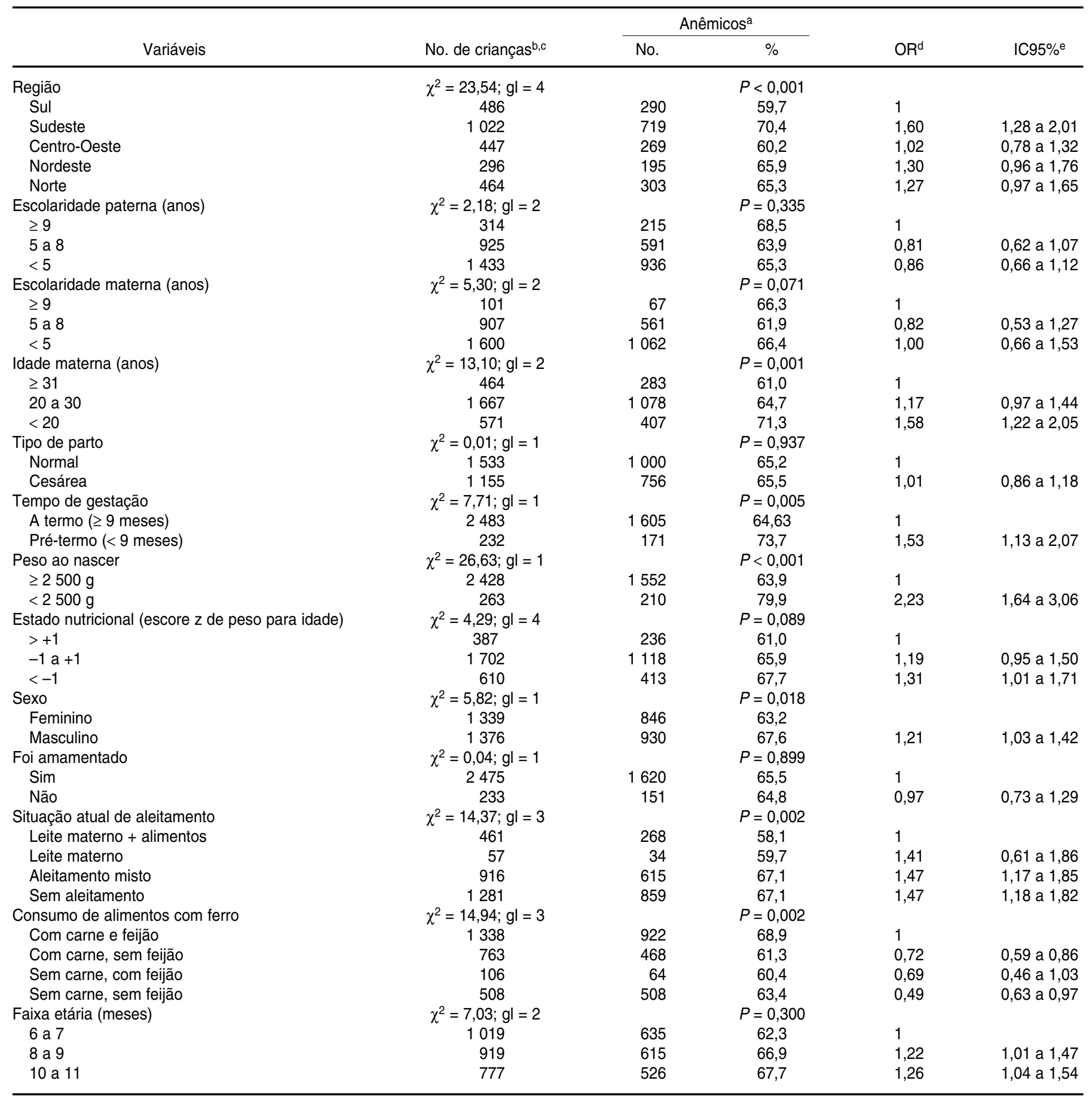

anemia: hemoglobina $<11 \mathrm{~g} / \mathrm{dL}$.

b $O$ total de crianças pode mudar para algumas variáveis em função da ausência de informações.

${ }^{c} \mathrm{Gl}=$ graus de liberdade.

d Razão de produtos cruzados (odds ratio).

e Intervalo de confiança de $95 \%$.

etapa, observou-se que o sexo masculino foi fator de risco para a anemia, enquanto que a idade da criança não se mostrou significativa (tabela 2).
A figura 2 mostra um esquema simplificado dos níveis hierárquicos e de como os vários blocos vão se compondo após a exclusão das variáveis que não mostraram associação após ajuste para os fatores de confusão. Tomando-se como categorias de referência a Região Sul, a escolaridade 
TABELA 2. Análise múltipla para fatores de risco de anemia em crianças de 6 a 12 meses conforme modelo com seleção hierárquica de variáveis, Brasil, 2000ª

\begin{tabular}{|c|c|c|c|c|c|c|c|c|c|c|}
\hline \multirow[b]{2}{*}{ Variáveis } & \multicolumn{2}{|c|}{$1^{\mathrm{a}}$ etapa } & \multicolumn{2}{|c|}{$2^{\mathrm{a}}$ etapa } & \multicolumn{2}{|c|}{$3^{\mathrm{a}}$ etapa } & \multicolumn{2}{|c|}{$4^{\mathrm{a}}$ etapa } & \multicolumn{2}{|c|}{$5^{\mathrm{a}}$ etapa } \\
\hline & OR & IC95\% & OR & IC95\% & OR & IC95\% & OR & IC95\% & OR & IC95\% \\
\hline \multicolumn{11}{|l|}{ Região } \\
\hline Sudeste & 1,60 & 1,28 a 2,01 & 1,55 & 1,23 a 1,96 & 1,60 & 1,27 a 2,00 & 1,55 & 1,23 a 1,96 & 1,57 & 1,25 a 1,99 \\
\hline Centro-Oeste & 1,02 & 0,78 a 1,32 & 1,02 & 0,78 a 1,33 & 1,00 & 0,77 a 1,30 & 1,04 & 0,79 a 1,36 & 1,03 & 0,78 a 1,36 \\
\hline Nordeste & 1,30 & 0,96 a 1,76 & 1,31 & 0,96 a 1,76 & 1,28 & 0,94 a 1,73 & 1,25 & 0,92 a 1,71 & 1,24 & 0,91 a 1,70 \\
\hline$\geq 9$ & & & 1 & & & & & & & \\
\hline 5 a 8 & & & 1,22 & 0,79 a 1,90 & $\mathrm{E}$ & & & & & \\
\hline$<5$ & & & 0,99 & 0,64 a 1,54 & $\mathrm{E}$ & & & & & \\
\hline \multicolumn{11}{|l|}{ Idade materna (anos) } \\
\hline$\geq 31$ & & & & & 1 & & 1 & & 1 & \\
\hline 20 a 30 & & & & & 1,21 & 0,98 a 1,50 & 1,24 & 0,99 a 1,54 & 1,23 & 0,99 a 1,54 \\
\hline$<20$ & & & & & 1,60 & 1,23 a 2,08 & 1,60 & 1,22 a 2,08 & 1,58 & 1,21 a 2,07 \\
\hline$\geq 2500$ & & & & & & & 1 & & 1 & \\
\hline$<2500$ & & & & & & & 2,01 & 1,42 a 2,85 & 2,09 & 1,48 a 2,95 \\
\hline \multicolumn{11}{|l|}{$\begin{array}{l}\text { Estado nutricional } \\
\text { (escore } z \text { de peso para altura) }\end{array}$} \\
\hline$>+1$ & & & & & & & 1 & & & \\
\hline$-1-+1$ & & & & & & & 1,17 & 0,93 a 1,47 & $\mathrm{E}$ & \\
\hline$<-1$ & & & & & & & 1,12 & 0,85 a 1,48 & $\mathrm{E}$ & \\
\hline \multicolumn{11}{|l|}{ Situação atual de aleitamento } \\
\hline Aleitamento materno + alimentos & & & & & & & 1 & & 1 & \\
\hline Aleitamento materno & & & & & & & 1,13 & 0,62 a 2,04 & 1,13 & 0,63 a 2,05 \\
\hline Aleitamento misto & & & & & & & 1,42 & 1,12 a 1,81 & 1,40 & 1,10 a 1,78 \\
\hline Sem aleitamento & & & & & & & 1,30 & 1,04 a 1,63 & 1,28 & 1,02 a 1,61 \\
\hline \multicolumn{11}{|l|}{ Consumo alimentos fonte de ferro } \\
\hline \multicolumn{11}{|l|}{ Sexo } \\
\hline Feminino & & & & & & & & & 1 & \\
\hline Masculino & & & & & & & & & 1,24 & 1,06 a 1,46 \\
\hline
\end{tabular}

a $\mathrm{OR}=$ razão de produtos cruzados (odds ratio); IC95\% = intervalo de confiança de 95\%. $\mathrm{E}=$ excluídos ( $P$ de Wald $>0,05$ na etapa de entrada na análise múltipla).

materna $\geq 9$ anos, a idade materna $\geq 31$ anos, o tempo de gestação a termo, o peso ao nascer maior ou igual a 2500 $\mathrm{g}$, o escore $\mathrm{Z}$ de peso para idade $>+1$, o aleitamento materno complementado com outros alimentos, o consumo de carne e de feijão, a faixa etária de 6 a 7 meses e o sexo feminino, a análise múltipla identificou os seguintes fatores de risco para anemia: morar na Região Sudeste, idade materna inferior a
20 anos, baixo peso ao nascer, não receber leite materno ou estar em aleitamento misto, e sexo masculino.

\section{DISCUSSÃO}

O presente estudo sugere que a prevalência de anemia ferropriva é alta em todo o Brasil para as crianças de 6 a 12 meses de idade atendidas em unidades básicas de saúde. Verificouse a maior prevalência de anemia entre as crianças do Sudeste $(70,4 \%)$ e a menor prevalência no Sul $(59,7 \%)$, com valor semelhante ao observado por Neuman et al. (18). Apesar de a Região Sudeste ser a mais rica do país, levanta-se a hipótese de que, com a maior inserção das mães no mercado de trabalho e com o término da licença-maternidade, a criança fique 
FIGURA 2. Variáveis associadas à anemia em crianças de 6 a 12 meses conforme modelo hierárquico, Brasil, 2000

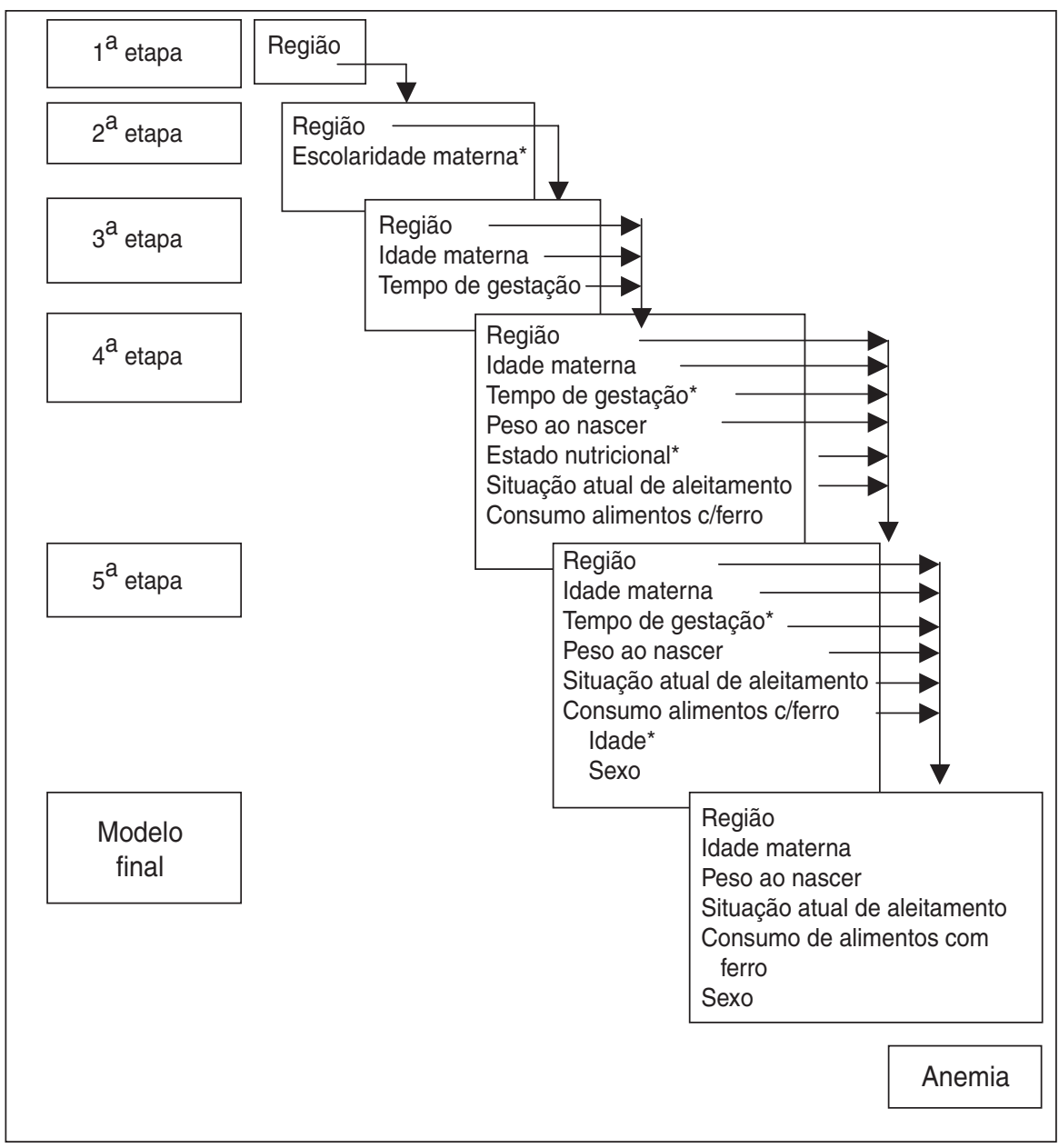

${ }^{a} \mathrm{O}$ asterisco indica perda de significância.

aos cuidados de pessoas menos qualificadas para o seu trato, favorecendo o aumento na prevalência de anemia. Os resultados parciais por localidade para a presente amostra revelaram dados homogêneos (19-21). As pesquisas realizadas em outros municípios brasileiros também têm encontrado resultados semelhantes: 60,9\% em Goiânia, Estado de Goiás (22); 60\% em Fortaleza, Estado do Ceará (23); 61,8\% em Recife, Estado de Pernambuco (24); $54 \%$ em Criciúma, Estado de Santa Catarina (18); e 47,8\% em Porto Alegre, Estado do Rio Grande do Sul (6).

Não foram encontradas diferenças entre as proporções de anêmicos quando consideradas as variáveis es- colaridade materna e paterna. Em relação à associação entre escolaridade materna e anemia, estudos em diferentes países apontam resultados contraditórios. No Brasil, Monteiro et al. (1), em estudo sobre a tendência secular da anemia nos inquéritos de 1984 e 1985 e 1995 e 1996, com amostra representativa da população entre 0 e 59 meses residente no município de São Paulo, verificaram um aumento na prevalência da anemia, apesar da melhoria nos níveis de renda familiar e escolaridade materna. Em contrapartida, Male et al. (25), em estudo europeu de coorte prospectivo, encontraram associação entre maior escolaridade materna e menor risco de anemia ferropriva em crianças com 12 meses de idade.

Neste estudo, escolaridade materna e paterna não se associaram com aumento de risco para anemia. Este achado pode ser decorrente da grande proporção de pais e mães com baixa escolaridade, caracterizando uma população de certa forma homogênea neste aspecto, corroborando achados de outras pesquisas que também trabalharam com populações de baixa renda $(22,26)$.

Foi observada uma associação significativa entre a idade materna e a anemia, com maior prevalência da doença entre os filhos de mães adolescentes. Quanto a esse aspecto, deve-se considerar a maior probabilidade que as mães adolescentes têm de conceber filhos com baixo peso, fator diretamente ligado à menor reserva de hemoglobina. Também foi verificada associação entre a idade materna e a introdução de alimentos complementares, como em outros estudos $(18,27)$. Tal fato poderia, em parte, ser explicado pela maior dificuldade das mães jovens em alimentar a criança, fazendo uma introdução inadequada da alimentação complementar, uma vez que somente os fatores fisiológicos maternos, relacionados às próprias necessidades da adolescente durante o período gestacional, dificilmente influenciariam os níveis de hemoglobina no $2^{\circ}$ semestre de vida da criança.

Houve uma maior prevalência de anemia nas crianças que nasceram com baixo peso, dado semelhante ao encontrado por outros pesquisadores $(21,28-30)$ que advertiram sobre a importância da atenção pré-natal no sentido de reduzir a incidência de peso inadequado ao nascer. Além disso, o estado nutricional inadequado (escore $Z$ de peso para idade $<-1$ ) representou um fator de risco para a anemia quando analisado sem exclusão dos fatores de confusão, corroborando estudos anteriores $(28,31,32)$, apesar de terem sido encontrados também dados discordantes $(33,34)$. Alguns estudos sugerem que a dieta das crianças tem melhorado apenas quantitativamente, e não qualitativamente, motivo pelo qual tem sido registrada uma tendência à diminuição da prevalência de 
desnutrição simultaneamente à elevação na prevalência da anemia (35). Apesar de não ter sido observada uma associação entre a amamentação e a anemia (provavelmente porque o aleitamento materno exclusivo ou predominante se restringe mais ao $1^{\circ}$ semestre), verificou-se que o aleitamento materno no $2^{\circ}$ semestre, complementado com outros alimentos, promove uma melhor situação com relação à anemia. Tal observação reforça a importância da manutenção do aleitamento materno no $1^{\circ}$ ano de vida.

A alta prevalência de anemia sugere também que a alimentação das crianças pesquisadas não é suficiente para suprir as necessidades de ferro, fato que possivelmente deva ser creditado à qualidade da alimentação oferecida, à falta de alimentos fortificados, ou à restrição na quantidade de alimentos decorrente da pequena capacidade estomacal da criança. Apesar disso, o consumo de alimentos fontes de ferro apresentou uma associação negativa com a anemia, sugerindo que, apesar do efeito protetor, são necessários estudos que avaliem o consumo alimentar quantitativamente.

Neste estudo, a anemia foi maior nos meninos $(P=0,018$, análise bivariada), achado consistente com a maior parte dos registros da literatura. A maior prevalência de anemia entre meninos é atribuída ao maior ganho de peso, às diferenças no consumo alimentar, ao aumento da atividade de eritropoese durante a vida fetal, às menores reservas, maiores perdas intestinais e menor absorção de ferro, ao maior número de episódios de infecção e ao menor tempo de amamentação por parte dos meninos em relação às meninas $(36,37)$.

A anemia, como outras doenças, é um problema de saúde com fatores determinantes múltiplos e complexos, que interagem para o seu desenvolvimento, motivo pelo qual tem sido estudada por meio de modelo com seleção hierarquizada. $\mathrm{O}$ presente trabalho partiu de um modelo conceitual que considera as causas distais, representadas pelo contexto social e cultural onde se situa a criança, assim como os fatores intermediários e proximais. Vários dos resultados encontrados utilizando-se esse método estão de acordo com outros estudos. Por exemplo, a escolaridade materna perdeu a significância na análise multivariada, conforme relatos anteriores $(22,38)$. Da mesma forma, o peso ao nascer inferior a $2500 \mathrm{~g}$ implicou uma probabilidade duas vezes maior de anemia, de modo consistente com os resultados encontrados por Uchimura et al. (19). Ainda, os riscos constatados para os diferentes tipos de aleitamento foram semelhantes aos encontrados por Monteiro et al. (1).
O consumo de carnes representou um fator de proteção. Outros estudos também comprovam o efeito protetor da carne $(22,34)$. É preciso observar que, para que a carne se torne um fator de proteção e possibilite um maior aporte de ferro, ela deve ser disponibilizada em quantidades superiores a $90 \mathrm{~g}$ por dia $(39,40)$. Isso dificulta sua incorporação na dieta do lactente, pois, para tal, seria necessário triturar a carne. Tal prática deveria ser muito bem explicada para a mãe, já que a liquidificação dos demais alimentos não é recomendada. O custo da incorporação diária desse alimento na dieta da criança é outro fator a ser considerado. Em suma, as baixas concentrações médias de hemoglobina e a alta proporção de crianças anêmicas observadas no presente estudo indicam a necessidade de enfatizar, nos programas de pré-natal e puericultura do Brasil, medidas de intervenção e controle desse distúrbio nutricional. Os fatores de risco identificados no presente estudo podem orientar tais ações, que devem privilegiar os grupos de maior risco, como são os nascidos de baixo peso e os filhos de mães adolescentes.

Agradecimentos. A pesquisa foi financiada pela Fundação de Amparo à Pesquisa do Estado de São Paulo (FAPESP, processo 1996/06886-3).

\section{REFERÊNCIAS}

1. Monteiro CA, Szarfarc SC, Mondini L. Tendência secular da anemia na infância na cidade de São Paulo (1984-1996). Rev Saude Publica. 2000;34(6):62-72.

2. Finch CA, Cook JD. Iron deficiency. Am J Clin Nutr. 1984;39(3):471-7.

3. Brasil, Ministério da Saúde. A saúde no Brasil. Brasília: Ministério da Saúde, Assessoria de Comunicação Social; 1997.

4. De Maeyer E, Adiels-Tegman M. The prevalence of anaemia in the world. World Health Stat Q. 1985;38(3):302-16.

5. Souza SB, Szarfarc SC, Souza JMP. Anemia no primeiro ano de vida em relação ao aleitamento materno. Rev Saude Publica. 1997;31(1):15-20.

6. Silva LSM, Giugliani ERJ, Aerts DRGC. Prevalência e determinantes de anemia em crianças de Porto Alegre, RS, Brasil. Rev Saude Publica. 2001;35(1):66-73.
7. Travé TD, Vélaz LD. Prevalencia de la deficiencia de hierro en lactentes sanos de 12 meses de edad. An Esp Pediatr. 2002;57(3): 209-14.

8. Fuchs SC, Victora CG, Fachel J. Modelo hierarquizado: uma proposta de modelagem aplicada à investigação de fatores de risco para diarréia grave. Rev Saude Publica. 1996;30(2): 168-78.

9. O Brasil e suas regiões. Disponível em: http:// www.brasilchannel.com.br/regioes/. Acessado em $1^{\circ}$ de novembro de 2004.

10. Von Shenck H, Falkensson M, Lundberg B. Evaluation of "Hemocue," a new device for determining hemoglobin. Clin Chem. 1986; 32(3):526-9.

11. Cohen A, Friedman J. HemoCue system for hemoglobin measurement, evaluation in anemic and nonanemic children. Am J Clin Pathol. 1988;90(3):302-5.
12. Be WKM, Kerkkamp HEM, Booij LHDJ. Hemocue-a new haemoglobinometer in the clinic. Eur J Anaesthesiol. 1991;8(1):55-8.

13. Thomas-Hudson M, Bingham KC, Simmons WK. An evaluation of the HemoCue for measuring hemoglobin in field studies in Jamaica. Bull World Health Org. 1994;73(3):423-6.

14. Organización Mundial de la Salud. Lucha contra la anemia nutricional, especialmente contra la carencia de hierro. Genebra: OMS; 1975. (Series de Informes Tecnicos, 580).

15. Rios E, Neuhauser L, Margen S, Melnick V. Accuracy of mothers' responses to questions about breast-feeding practices. Food Nutr Bull. 1992;14(2):115-8.

16. Silva LC, Fuentelsaz C, Amador M. Características de la introducción de alimentos al lactante en Cuba. Bol Oficina Sanit Panam. 1993;114(5):407-14. 
17. Stata Corp. Stata. Statistical Software. Release 70. College Station, TX: Stata Corporation; 2001.

18. Neuman NA, Tanaka OY, Szarfarc SC, Guimarães PRV, Victora CG. Prevalência e fatores de risco para anemia no Sul do Brasil. Rev Saude Publica. 2000;34(1):56-63.

19. Uchimura TT, Szarfarc SC, Latorre MRD, Uchimura NS, Souza SB. Anemia e peso ao nascer. Rev Saude Publica. 2003;36(4):397-403.

20. Alberico APM, Veiga GV, Baião MR, Santos MMAS, Souza SB, Szarfarc SC. Iron deficiency anaemia in infants attended at municipal primary health care centers in Rio de JaneiroBrazil. Nutr Food Sci. 2003;33(2):50-5.

21. Silva DG, Franceschini SCC, Priore SE, Ribeiro SMR, Szarfarc SC, Souza SB, et al. Anemia ferropriva em crianças de 6 a 12 meses atendidas na rede pública de saúde do município de Viçosa, Minas Gerais. Rev Nutr. 2002;15(3):301-8.

22. Hadler MCCM, Juliano Y, Sigulem DM. Anemia do lactente: etiologia e prevalência. J Pediatr (Rio J). 2002;78(4):321-6.

23. Soares NT, Guimarães ARP, Sampaio HAC, Almeida PC, Coelho RR. Estado nutricional de lactentes em áreas periféricas de Fortaleza. Rev Nutr. 2000;13(2):99-106.

24. Osório MM, Lira PIC, Batista-Filho M, Ashworth A. Prevalence of anemia in children 6-59 months old in the state of Pernambuco, Brazil. Rev Panam Salud Publica. 2001;10(2): 101-7.

25. Male C, Persson LA, Freeman V, Guerra A, van't Hof MA, Haschke F, Euro-Growth Iron Study Group. Prevalence of iron deficiency in 12-mo-old infants from 11 European areas and influence of dietary factors on iron status
(Euro-Growth study). Acta Paediatr. 2001;90 (5):492-8.

26. Sigulem DM, Tudisco ES, Goldemberg P, Athaide MMM, Vaisman E. Anemia ferropriva em crianças no município de São Paulo. Rev Saude Publica. 1978;12(2):168-78.

27. Marchioni DML, Latorre MRDO, Szarfarc SC, Souza SB. Complementary feeding: study on prevalence of food intake in two health centers of São Paulo City. Arch Latinoam Nutr. 2001;51(2):161-6.

28. Torres MAA, Sato K, Queiroz SS. Anemia em crianças menores de dois anos atendidas nas Unidades Básicas de Saúde do Estado de São Paulo. Rev Saude Publica. 1994;28(4):290-4.

29. Dewey KG, Cohen RJ, Rivera LL, Brown KH. Effects of age of introduction of complementary foods on iron status of breast-fed infants in Honduras. Am J Clin Nutr. 1998;67(5): 878-84.

30. Lartey A, Manu A, Brown KH, Dewey KG. Predictors of micronutrient status among sixto twelve-month-old breast-fed Ghanaian infants. J Nutr. 2000;130(2):199-207.

31. Schmitz BAS, Picanço MR, Aquino KKNC, Bastos J, Giorgini E, Cardoso R, et al. Prevalência de desnutrição e anemia em préescolares de Brasília (Brasil). Pediatr Mod. 1998;34(4):155-64.

32. Brunken GS, Guimarães LV, Fisberg M. Anemia em crianças menores de 3 anos que freqüentam creches públicas em período integral. J Pediatr (Rio J). 2002;78(1):50-6.

33. Rodrigues CRM, Motta SS, Cordeiro AA, Lacerda EM, Reichenheim ME. Prevalência de anemia ferropriva e marcadores de risco associados em crianças entre 12 e 18 meses de idade atendidas nos ambulatórios do Instituto de Puericultura e Pediatria Martagão Gesteira. J Pediatr (Rio J). 1997;73(3):189-94.

34. Devincenzi UM. Evolução dos níveis de hemoglobina: estudo em crianças menores de dois anos acompanhadas pelo Projeto Favela [tese]. São Paulo: Universidade Federal de São Paulo (UNIFESP)/Escola Paulista de Medicina; 2004.

35. Batista Filho M, Rissin A. A transição nutricional no Brasil: tendências regionais e temporais. Cad Saude Publica. 2003;19(Sup 1): S181-91.

36. Dömellof M, Lönnerdal B, Dewey KG, Cohen RJ, Rivera LL, Hernell O. Sex differences in iron status during infancy. Pediatrics. 2002; 110(3):545-52.

37. Venâncio SI. Determinantes individuais e contextuais do aleitamento materno exclusivo nos primeiros seis meses de vida em cento e onze municípios do Estado de São Paulo [tese]. São Paulo: Faculdade de Saúde Pública, Universidade de São Paulo (USP); 2002.

38. Compri PC. Anemia ferropriva e os serviços de atenção primária à saúde: fatores de risco e qualidade de atendimento [tese]. São Paulo: Universidade Federal de São Paulo (UNIFESP)/Escola Paulista de Medicina; 2004.

39. Layrisse M, Garcia-Casal MN. Strategies for the prevention of iron deficiency through foods in the household. Nutr Rev. 1997;55(6): 233-9.

40. World Health Organization. Child health and development. Evidence for the ten steps to successful breast-feeding. Genebra: WHO; 1998

Manuscrito recebido em 28 de janeiro de 2004. Aceito em versão revisada em 31 de agosto de 2004.

ABSTRACT Objective. To estimate the prevalence of anemia and to determine associated risk factors among infants receiving routine health care in public clinics in Brazil.

Method. This cross-sectional study included 2715 infants between 6 and 12 months Risk factors for anemia among 6- to 12-month-old children in Brazil

old in 12 cities, in all five of the geographic regions of Brazil. Information regarding the child and its feeding habits was obtained from the mother or other caregiver, using a questionnaire. Nutritional status was determined based on height and weight measurements. The hemoglobin concentration was measured using the HemoCue portable hemoglobinometer. Anemia was defined as hemoglobin $<11 \mathrm{~g} / \mathrm{dL}$. The infants' eating habits were assessed based on what they were eating around the time of the questionnaire interviews. The association between anemia and the different variables was evaluated through bivariate analysis, followed by multiple logistic regression using a hierarchical selection model.

Results. The prevalence of anemia for the entire group was 65.4\%. Multiple regression analysis identified the following risk factors for anemia: living in the Southeastern Region of Brazil (odds ratio $(\mathrm{OR})=1.57,95 \%$ confidence interval $(95 \% \mathrm{CI})=1.25$ 1.99), maternal age $<20$ years $(\mathrm{OR}=1.58,95 \% \mathrm{CI}=1.21-2.07)$, birthweight $<2500 \mathrm{~g}$ $(\mathrm{OR}=2.09,95 \% \mathrm{CI}=1.48-2.95)$, not being breast-fed $(\mathrm{OR}=1.28,95 \% \mathrm{CI}=1.02-1.61)$, receiving both breast milk and other foods $(\mathrm{OR}=1.40,95 \% \mathrm{CI}=1.10-1.78)$, and male gender $(\mathrm{OR}=1.24,95 \% \mathrm{CI}=1.06-1.46)$.

Conclusions. The high proportion of anemic children indicates the need to emphasize, in prenatal and infant health programs, intervention measures for anemia control. Our results could guide these measures, focusing on the groups at greatest risk, such as low birthweight babies and the children of adolescent mothers.

Keywords: Anemia, iron-deficiency; infant nutrition; child nutrition; risk factors; epidemiologic studies. 